\title{
The association between kidney function and major bleeding in older adults with atrial fibrillation starting warfarin treatment: population based observational study
}

\author{
Min Jun, ${ }^{1}$ Matthew T James, ${ }^{1,2}$ Braden J Manns, ${ }^{1,2}$ Robert R Quinn, ${ }^{1,2}$ Pietro Ravani,, 2 \\ Marcello Tonelli, ${ }^{1}$ Vlado Perkovic, ${ }^{3}$ Wolfgang C Winkelmayer, ${ }^{4}$ Zhihai Ma, ${ }^{1}$ \\ Brenda R Hemmelgarn, ${ }^{1,2}$ for the Alberta Kidney Disease Network
}

'Department of Medicine,

Division of Nephrology,

University of Calgary, AB,

Canada, T2N 2T9

${ }^{2}$ Department of Community Health Sciences, University of

Calgary, Calgary, AB, Canada

${ }^{3}$ The George Institute for Global Health, The University of Sydney, Sydney, Australia

${ }^{4}$ Section of Nephrology,

Baylor College of Medicine,

Houston, TX, USA

Correspondence to:

B Hemmelgarn

Brenda.Hemmelgarn@

albertahealthservices.ca

Additional material is published online only. To view please visit the journal online (http://

dx.doi.org/10.1136/BMJ.h246)

Cite this as: $B M J$ 2015;350:h246 doi: 10.1136/bmj.h246

Accepted: 5 December 2014

\section{ABSTRACT}

OBJECTIVE

To determine rates of major bleeding by level of kidney function for older adults with atrial fibrillation starting warfarin.

DESIGN

Retrospective cohort study.

SETTING

Community based, using province wide laboratory and administrative data in Alberta, Canada.

\section{PARTICIPANTS}

12403 adults aged 66 years or more, with atrial fibrillation who started warfarin treatment between 1 May 2003 and 31 March 2010 and had a measure of kidney function at baseline. Kidney function was estimated using the Chronic Kidney Disease Epidemiology Collaboration equation and participants were categorised based on estimated glomerular filtration rate (eGFR): $\geq 90,60-89,45-59,30-44$, $15-29,<15 \mathrm{ml} / \mathrm{min} / 1.73 \mathrm{~m}^{2}$. We excluded participants with end stage renal disease (dialysis or renal transplant) at baseline.

\section{MAIN OUTCOME MEASURES}

Admission to hospital or visit to an emergency department for major bleeding (intracranial, upper and lower gastrointestinal, or other).

RESULTS

Of 12403 participants, $45 \%$ had an eGFR $<60 \mathrm{ml} / \mathrm{min} /$ $1.73 \mathrm{~m}^{2}$. Overall, 1443 (11.6\%) experienced a major bleeding episode over a median follow-up of 2.1 (interquartile range: $1.0-3.8$ ) years. During the first 30 days of warfarin treatment, unadjusted and adjusted

\section{WHAT IS ALREADY KNOWN ON THIS TOPIC}

Although the risk of bleeding is considerably higher among patients who require dialysis than in the general population, there are limited data about the bleeding risk associated with warfarin treatment in people with different stages of chronic kidney disease

\section{WHAT THIS STUDY ADDS}

Reduced kidney function, in patients not requiring dialysis, was associated with an increased risk of major bleeding among older adults with atrial fibrillation starting warfarin

The risk of warfarin treatment should be weighed against the potential benefits based on the presence of comorbidities and bleeding risk among patients with reduced kidney function (for example, $<60 \mathrm{ml} / \mathrm{min} / 1.73 \mathrm{~m}^{2}$ ), and particularly in those with very reduced kidney function and during the first 30 days of treatment

rates of major bleeding were higher at lower eGFR ( $P$ for trend $<0.001$ and 0.001 , respectively). Adjusted bleeding rates per 100 person years were 63.4 (95\% confidence interval 24.9 to 161.6$)$ in participants with eGFR $<15 \mathrm{ml} / \mathrm{min} / 1.73 \mathrm{~m}^{2}$ compared with 6.1 (1.9 to 19.4) among those with eGFR $>90 \mathrm{ml} / \mathrm{min} / 1.73 \mathrm{~m}^{2}$ (adjusted incidence rate ratio 10.3, 95\% confidence interval 2.3 to 45.5). Similar associations were observed at more than 30 days after starting warfarin, although the magnitude of the increase in rates across eGFR categories was attenuated. Across all eGFR categories, adjusted rates of major bleeding were consistently higher during the first 30 days of warfarin treatment compared with the remainder of follow-up. Increases in major bleeding rates were largely due to gastrointestinal bleeding (3.5-fold greater in eGFR $<15 \mathrm{ml} / \mathrm{min} / 1.73 \mathrm{~m}^{2}$ compared with $\geq 90 \mathrm{ml} / \mathrm{min} /$ $1.73 \mathrm{~m}^{2}$ ). Intracranial bleeding was not increased with worsening kidney function.

\section{CONCLUSIONS}

Reduced kidney function was associated with an increased risk of major bleeding among older adults with atrial fibrillation starting warfarin; excess risks from reduced eGFR were most pronounced during the first 30 days of treatment. Our results support the need for careful consideration of the bleeding risk relative to kidney function when assessing the risk-benefit ratio of warfarin treatment in people with chronic kidney disease and atrial fibrillation, particularly in the first 30 days of treatment.

\section{Introduction}

Chronic kidney disease is a global public health concern and is associated with increased morbidity, mortality, and healthcare costs, in part related to higher rates of cardiovascular disease, including stroke. ${ }^{12}$ The prevalence of atrial fibrillation increases not only with age but also with declining kidney function and is almost fourfold higher among patients with an estimated glomerular filtration rate (eGFR) $<30 \mathrm{ml} / \mathrm{min} /$ $1.73 \mathrm{~m}^{2}$ and up to 13 -fold higher in patients who require haemodialysis compared with the general population. ${ }^{3}$ Atrial fibrillation is associated with an increased risk of stroke, ${ }^{45}$ and warfarin is commonly prescribed to reduce this risk in the general population. Although the risk of bleeding is considerably higher among patients who require dialysis than in the general population, there are limited data about the bleeding risk associated with warfarin treatment in people with different stages of chronic kidney disease. ${ }^{6-8}$ 
Safety data about rates of major bleeding from warfarin treatment have been reported by randomised trials ${ }^{910}$ and a small number of studies using administrative data $^{1112}$ including people with atrial fibrillation. However, these rates from randomised trials often do not reflect the real world clinical setting, whereas the population based studies to date are limited by their lack of baseline kidney function, assessment of prevalent rather than incident warfarin use, and potential for selection bias, particularly confounding by indication. ${ }^{11}{ }^{12}$ In addition, recent evidence in the general population suggests that the risk of bleeding is highest in the first 30 days of warfarin treatment, thus the association between kidney function and bleeding risk may be particularly pronounced during this period. ${ }^{13}$

We carried out a population based study of people with atrial fibrillation who started warfarin treatment to determine if rates of major bleeding varied by level of kidney function and duration of treatment (first 30 days and after 30 days).

\section{Methods}

Identification of study cohort

Warfarin use and history of atrial fibrillation-We carried out a retrospective cohort study using laboratory and administrative data from Alberta, Canada. ${ }^{14}$ Albertans are eligible for subsidised prescription drug coverage at age 65; therefore to allow for a one year baseline assessment period, the study population included Alberta residents aged 66 years or more who had a recorded history of atrial fibrillation (international classification of diseases, ninth revision code 427.31/2; 10th revision code I48), ${ }^{15-17}$ and started warfarin between 1 May 2003 and 31 March 2010. The index date was defined as the date of the first dispensed warfarin prescription after the participant's 66th birthday, in those with no prescription in the previous year. We defined continuous warfarin use as two or more prescriptions within 180 days (in Alberta, prescriptions are typically dispensed for 90 days). If 180 days or more elapsed between warfarin prescriptions, we considered the participant to have stopped warfarin treatment.

Assessment of kidney function-Eligible participants were those with one or more outpatient serum creatinine measurements within the one year before, or 90 days after, the index date. ${ }^{14}$ We estimated baseline glomerular filtration rate (eGFR) using the Chronic Kidney Disease Epidemiology Collaboration (CKD-EPI) equation $^{18}$ and grouped participants into the KDIGO (Kidney Disease: Improving Global Outcomes) eGFR categories: $\geq 90$ (reference group), 60-89, 45-59, 30-44, 15-29, and $<15 \mathrm{ml} / \mathrm{min} / 1.73 \mathrm{~m}^{2}{ }^{19}$ As we were interested in risk among the non-dialysis population we excluded participants with end stage renal disease, defined as having received chronic dialysis or renal transplantation at baseline. ${ }^{14}$

\section{Covariates}

We obtained information on personal characteristics and comorbid conditions from the administrative data files of the provincial health ministry. Aboriginal race or ethnicity was determined from First Nations status in the registry file. It was not possible to identify other racial or ethnic groups; however, more than $85 \%$ of the population of Alberta is white. ${ }^{20}$ Using validated algorithms, we identified the presence of diabetes mellitus ${ }^{21}$ and hypertension. ${ }^{22}$ We used the hospital discharge records and physician claims to calculate the $\mathrm{CHA}_{2} \mathrm{DS}_{2}$ VASc score (based on a previously validated algorithm), a risk score that estimates the risk of stroke in patients with atrial fibrillation. ${ }^{23}$ As our datasets did not provide information on alcohol use and we had data on international normalised ratios for only a small subset of the cohort, we calculated a modified HAS-BLED score (a risk score that estimates the risk of bleeding for patients taking anticoagulants for atrial fibrillation) without these variables, with a maximum score of 7 instead of 9. Proteinuria was measured by urine dipstick based on random outpatient spot urine measurements in the six month period before and after the index date and was defined as normal (urine dipstick negative), mild (urine dipstick trace or + ), heavy (urine dipstick ++ or more), and not measured (no outpatient urine dipstick measurement).

Other comorbid conditions based on the Deyo classification of Charlson comorbidities were identified using validated ICD ninth and 10th revision coding algorithms. ${ }^{24}$ We identified previous admissions to hospital involving bleeding within the three year period before the index date. From prescription drug records we obtained use of antiplatelet agents, non-steroidal anti-inflammatory drugs, and proton pump inhibitors, defined as one or more prescriptions within 120 days before the index date. We linked the six digit residential postal code for each participant to the 2001 or 2006 Canadian census (whichever was closest to the index date) using the postal code conversion file to determine the median neighbourhood household income fifth (levels 1 (lowest fifth) to 5 (highest fifth)) and location of residence (rural, urban, or undefined).

\section{Outcomes}

The outcome of interest was the first admission to hospital or visit to an emergency department for major bleeding, defined as an intracranial, upper or lower gastrointestinal, or other bleeding, based on a validated algorithm (see supplementary table 1 for list of the ICD-9 and ICD-10 codes). ${ }^{25}$ Participants were followed from their index date until the date of the major bleeding episode, end of warfarin treatment, death, out migration from the province, or study end (31 March 2011). In participants who stopped warfarin, we defined the date of the end of treatment as the date of the last warfarin prescription after the index date plus 90 days.

We used the results for international normalised ratio, available for a subset of the cohort only, to determine whether participants who experienced a major bleeding episode and had reduced kidney function were more likely to have an increased international normalised ratio (at the time of major bleeding). We assessed the proportion of participants with an international normalised ratio above the therapeutic range ( $>3)$, by eGFR 
categories. We used the highest result within a three day period of the bleeding episode (one day before, on the day of, and one day after the bleeding). We also sought to assess the severity of the major bleeding events based on the lowest haemoglobin result within a three day period of the bleeding episode (one day before, on the day of, and one day after the bleeding episode). In addition, among participants who experienced a gastrointestinal bleeding event, we determined the proportion who had undergone one or more diagnostic or therapeutic endoscopic procedures after seven days of the bleeding event, based on relevant procedure codes (see supplementary table 1 ).

\section{Statistical analyses}

We used Poisson regression to estimate the unadjusted and adjusted rates of major bleeding by category of eGFR. Based on a prespecified analysis plan, we estimated the unadjusted and adjusted rates of major bleeding in the first 30 days of warfarin treatment, as well as the period after the first 30 days. Rates, expressed per 100 person years, were adjusted for sociodemographic variables (sex, age, First Nations' status, region of residence, and neighbourhood income fifth), comorbidities (diabetes, hypertension, Charlson comorbidities (history of cancer, cerebrovascular disease, congestive heart failure, chronic obstructive pulmonary disease, dementia, metastatic solid tumour, myocardial infarction, mild liver disease, moderate or severe liver disease, paralysis, peptic ulcer disease, peripheral vascular disease, and rheumatic disease), previous admission to hospital for a bleeding episode, proteinuria (normal, mild, heavy, or not measured), and drug use (antiplatelet agents, non-steroidal anti-inflammatory drugs, and proton pump inhibitors). To assess the relative risk of major bleeding by eGFR category compared with the reference (eGFR $>90 \mathrm{ml} / \mathrm{min} / 1.73 \mathrm{~m}^{2}$ ) we determined incidence rate ratios and their $95 \%$ confidence intervals. To determine the association between kidney function and international normalised ratio above the therapeutic range $(>3)$ in participants who experienced a major bleeding event, we assessed the proportion with an international normalised ratio value $>3$, by eGFR categories. Based on the adjusted incidence rates of major bleeding in chronic kidney disease (defined as $<60 \mathrm{ml} / \mathrm{min} / 1.73 \mathrm{~m}^{2}$ ) and non-chronic kidney disease $\left(\geq 60 \mathrm{ml} / \mathrm{min} / 1.73 \mathrm{~m}^{2}\right)$, we determined the number needed to treat to harm in the first 30 days of warfarin treatment, as well as in the remainder of the study follow-up.

We performed several sensitivity analyses to confirm the robustness of our findings. Firstly, we repeated all analyses using $\geq 60 \mathrm{ml} / \mathrm{min} / 1.73 \mathrm{~m}^{2}$ as the reference group. Secondly, we repeated all analyses in participants who were admitted to hospital for a major bleeding episode only (that is, excluding those with the outcome event identified from visit to an emergency department without subsequent admission), as these would reflect the most severe cases. Thirdly, we included participants in whom an outpatient serum creatinine measurement was not available (categorised as chronic kidney disease status "missing") and compared their baseline characteristics with those of participants included in the final study cohort. Finally, we repeated all analyses, defining the end date of warfarin treatment (for participants who stopped warfarin before the study end) as the date of the last warfarin prescription plus 30 days, as some people may have stopped treatment earlier than the 90 day period used in the primary analysis. Statistical analyses were performed with STATA version 11.2 (STATA, TX). We considered a two sided $\mathrm{P}$ value less than 0.05 to be statistically significant.

\section{Results}

\section{Participant characteristics}

We identified 15319 participants aged 66 years or more with a history of atrial fibrillation who started warfarin treatment during the cohort enrolment period. We excluded 158 people who had end stage renal disease at the time of cohort entry. Of the remaining people, 12 403 had one or more outpatient serum creatinine measurements and were eligible for inclusion. The mean age of the cohort was 77 (SD 7.0) years and 49.3\% were women. Forty five per cent of study participants had an eGFR $<60 \mathrm{ml} / \mathrm{min} / 1.73 \mathrm{~m}^{2}$. Overall, the prevalence of cerebrovascular disease, heart failure, myocardial infarction, peripheral vascular disease, and previous admission to hospital for a bleeding episode increased with worsening baseline eGFR. Table 1 shows the baseline characteristics of the study participants by eGFR categories.

\section{Incidence of major bleeding}

Over a median follow-up of 2.1 years (interquartile range 1.0-3.8 years), 1443 participants (11.6\%) experienced a major bleeding episode. The overall incidence of major bleeding in the first 30 days of warfarin treatment was 15.2 (95\% confidence interval 13.0 to 17.9) per 100 person years, and after 30 days of warfarin treatment was 4.2 (4.0 to 4.4) per 100 person years (see supplementary figure 1$)$.

\section{Incidence rates of major bleeding by eGFR and follow-up period}

In the first 30 days of warfarin treatment the unadjusted and adjusted rates of bleeding increased with worsening levels of eGFR ( $P$ for trend $<0.001$ and 0.001 , respectively; fig 1 ). The adjusted rate of bleeding among those with $\mathrm{eGFR}<15 \mathrm{ml} / \mathrm{min} / 1.73 \mathrm{~m}^{2}$ was more than 10-fold higher than the reference group ( $\geq 90 \mathrm{ml} /$ $\min / 1.73 \mathrm{~m}^{2}$ ) during the first 30 days of treatment (63.4, 95\% confidence interval 24.9 to 161.6 per 100 person years $v 6.1,1.9$ to 19.4 per 100 person years, respectively; incidence rate ratio 10.33 , 95\% confidence interval 2.34 to 45.54; Table 2).

Similar associations were observed in the period after 30 days of warfarin treatment, where the unadjusted and adjusted rates of bleeding increased with decreasing levels of eGFR ( $P$ for trend $<0.001$ and 0.002 , respectively; fig 1 ). The magnitude of the 


\begin{tabular}{|c|c|c|c|c|c|c|}
\hline \multirow[b]{2}{*}{ Characteristics } & \multicolumn{6}{|c|}{$\mathrm{eGFR}\left(\mathrm{mL} / \mathrm{min} / 1.73 \mathrm{~m}^{2}\right)$} \\
\hline & $\geq 90(n=581)$ & $60-89(n=6140)$ & $45-59(n=3221)$ & $30-44(n=1820)$ & $15-29(n=586)$ & $<15(n=55)$ \\
\hline Women & $240(41.3)$ & $2797(45.5)$ & $1689(52.4)$ & $1022(56.1)$ & $342(58.3)$ & $24(43.6)$ \\
\hline Mean (SD) age (years) & $70.7(4.5)$ & $76.4(6.5)$ & $78.8(6.7)$ & $80.6(6.9)$ & $81.8(6.8)$ & $80.0(7.9)$ \\
\hline First Nations status & $8(1.3)$ & $45(0.7)$ & $17(0.5)$ & $10(0.5)$ & $2(0.3)$ & $0(0)$ \\
\hline \multicolumn{7}{|l|}{ Region of residence: } \\
\hline Rural & $129(22.2)$ & $1277(20.8)$ & $661(20.5)$ & $371(20.3)$ & $116(19.8)$ & $7(12.7)$ \\
\hline Urban & $448(77.1)$ & $4817(78.4)$ & $2531(78.5)$ & $1426(78.3)$ & $463(79.0)$ & $45(81.8)$ \\
\hline Unknown & $4(0.6)$ & $46(0.7)$ & $29(0.9)$ & $23(1.2)$ & $7(1.1)$ & $3(5.4)$ \\
\hline \multicolumn{7}{|l|}{ Income fifths: } \\
\hline 1 (lowest) & $124(21.3)$ & $1143(18.6)$ & 637 (19.7) & $362(19.8)$ & 105 (17.9) & $10(18.1)$ \\
\hline 2 & $107(18.4)$ & $1310(21.3)$ & $673(20.8)$ & $417(22.9)$ & $129(22)$ & $10(18.1)$ \\
\hline 3 & $107(18.4)$ & $1221(19.8)$ & $635(19.7)$ & $364(20)$ & $116(19.8)$ & $12(21.8)$ \\
\hline 4 & $115(19.7)$ & $1080(17.5)$ & $582(18)$ & $303(16.6)$ & $115(19.6)$ & $6(10.9)$ \\
\hline 5 (highest) & 109 (18.7) & $1202(19.5)$ & $583(18.1)$ & $300(16.4)$ & $100(17)$ & $14(25.4)$ \\
\hline Unknown & $19(3.2)$ & $184(3)$ & $111(3.4)$ & $74(4)$ & $21(3.5)$ & $3(5.4)$ \\
\hline Hypertension & $96(16.5)$ & $1025(16.6)$ & $495(15.3)$ & $218(11.9)$ & $47(8.0)$ & $6(10.9)$ \\
\hline Diabetes & $47(8.0)$ & $451(7.3)$ & $200(6.2)$ & $121(6.6)$ & $42(7.1)$ & $3(5.4)$ \\
\hline Cancer & $118(20.3)$ & 1100 (17.9) & $550(17.1)$ & $305(16.7)$ & $116(19.8)$ & $9(16.3)$ \\
\hline Cerebrovascular disease & $92(15.8)$ & $1150(18.7)$ & $713(22.1)$ & $387(21.26)$ & $143(24.4)$ & $12(21.8)$ \\
\hline Congestive heart failure & $181(31.1)$ & $2013(32.7)$ & $1367(42.4)$ & $1026(56.3)$ & $380(64.8)$ & $35(63.6)$ \\
\hline Chronic obstructive pulmonary disease & $238(40.9)$ & $1980(32.2)$ & $1059(32.8)$ & $680(37.3)$ & $232(39.5)$ & $29(52.73)$ \\
\hline Dementia & $28(4.8)$ & $355(5.7)$ & $208(6.4)$ & $177(9.7)$ & $71(12.1)$ & $4(7.2)$ \\
\hline Metastatic solid tumour & $30(5.1)$ & $184(3)$ & $69(2.1)$ & $49(2.6)$ & $19(3.2)$ & $2(3.6)$ \\
\hline Myocardial infarction & $130(22.3)$ & $1346(21.9)$ & $820(25.4)$ & $600(32.9)$ & $200(34.1)$ & $24(43.6)$ \\
\hline Mild liver disease & $14(2.4)$ & $80(1.3)$ & $47(1.4)$ & $43(2.3)$ & $10(1.7)$ & $1(1.8)$ \\
\hline Moderate or severe liver disease & $1(0.1)$ & $15(0.2)$ & $7(0.2)$ & $4(0.2)$ & $3(0.5)$ & $1(1.8)$ \\
\hline Paralysis & $18(3.1)$ & $198(3.2)$ & $89(2.7)$ & $50(2.7)$ & $11(1.8)$ & $1(1.8)$ \\
\hline Peptic ulcer disease & $38(6.5)$ & $263(4.2)$ & $139(4.3)$ & $93(5.1)$ & $36(6.1)$ & $2(3.6)$ \\
\hline Peripheral vascular disease & $72(12.3)$ & $654(10.6)$ & $384(11.9)$ & $281(15.4)$ & $114(19.4)$ & $14(25.4)$ \\
\hline Rheumatic disease & $42(7.2)$ & $247(4)$ & $124(3.8)$ & $95(5.2)$ & $26(4.4)$ & $2(3.6)$ \\
\hline Previous admission to hospital for bleeding & $31(5.3)$ & $237(3.8)$ & $164(5.0)$ & $107(5.8)$ & $43(7.3)$ & $7(12.7)$ \\
\hline \multicolumn{7}{|l|}{$\mathrm{CHA}_{2} \mathrm{DS}_{2}$-VASc score: ${ }^{*}$} \\
\hline 1 & $110(18.9)$ & $728(11.8)$ & $183(5.6)$ & $63(3.4)$ & $11(1.8)$ & $2(3.6)$ \\
\hline$\geq 2$ & $471(81.0)$ & $5412(88.1)$ & $3038(94.3)$ & $1757(96.5)$ & $575(98.1)$ & $53(96.3)$ \\
\hline \multicolumn{7}{|l|}{ Modified HAS-BLED score: ${ }^{+}$} \\
\hline 1 & $318(54.7)$ & $3276(53.3)$ & $1539(47.7)$ & $697(38.3)$ & $120(20.4)$ & $4(7.2)$ \\
\hline 2 & $201(34.6)$ & $2220(36.1)$ & $1196(37.1)$ & $730(40.1)$ & $282(48.1)$ & $29(52.7)$ \\
\hline 3 & $51(8.7)$ & $563(9.1)$ & $403(12.5)$ & $325(17.8)$ & $141(24.0)$ & $16(29.0)$ \\
\hline 4 & $11(1.8)$ & $74(1.2)$ & $78(2.4)$ & $57(3.1)$ & $39(6.6)$ & $4(7.2)$ \\
\hline 5 & $0(0)$ & $7(0.1)$ & $5(0.1)$ & $11(0.6)$ & $4(0.6)$ & $2(3.6)$ \\
\hline \multicolumn{7}{|l|}{ Proteinuria: } \\
\hline Normal & $321(55.2)$ & $3304(53.8)$ & $1702(52.8)$ & $934(51.3)$ & $267(45.5)$ & $14(25.4)$ \\
\hline Mild & $79(13.6)$ & $828(13.4)$ & $469(14.5)$ & $316(17.3)$ & $119(20.3)$ & $14(25.4)$ \\
\hline Heavy & $25(4.3)$ & $261(4.2)$ & $201(6.2)$ & $157(8.6)$ & $101(17.2)$ & $23(41.8)$ \\
\hline Not measured & $156(26.8)$ & $1747(28.4)$ & $849(26.3)$ & $413(22.6)$ & $99(16.8)$ & $4(7.2)$ \\
\hline \multicolumn{7}{|l|}{ Drug use: } \\
\hline Antiplatelet agent & $5(0.8)$ & $74(1.2)$ & $46(1.4)$ & $31(1.7)$ & $12(2.0)$ & $0(0)$ \\
\hline Non-steroidal anti-inflammatory drugs & $82(14.1)$ & $841(13.7)$ & $479(14.8)$ & $258(14.1)$ & $87(14.8)$ & $5(9.0)$ \\
\hline Proton pump inhibitors & $178(30.6)$ & $1832(29.8)$ & $1102(34.2)$ & $719(39.5)$ & $249(42.4)$ & $19(34.5)$ \\
\hline \multicolumn{7}{|c|}{$\begin{array}{l}\text { *No participant had a } \mathrm{CHA}_{2} \mathrm{DS} \mathrm{S}_{2} \text {-VASc (a risk score that estimates the risk of stroke in patients with atrial fibrillation; } 1 \text { point each is given in the presence of the included risk factors: congestive } \\
\text { heart failure, hypertension, diabetes mellitus, vascular disease, age } 65-74 \text { years, and female sex; } 2 \text { points are given in the presence of the included risk factors: age } \geq 75 \text { years and previous } \\
\text { stroke, transient ischaemic attack, or thromboembolism) score of } 0 \text {. } \\
\text { }{ }^{+} \text {Calculated as a modified HAS-BLED (a risk score that estimates the risk of major bleeding for patients taking anticoagulants for atrial fibrillation; in the original HAS-BLED score, } 1 \text { point each is } \\
\text { given in the presence of the included risk factors: hypertension, renal disease, liver disease, stroke, major bleeding, labile international normalised ratios, age } \geq 65 \text { years, concomitant } \\
\text { treatment with antiplatelet or non-steroidal anti-inflammatory drugs, and alcohol intake) score without alcohol and international normalised ratio values with a maximum score of } 7 \text {, instead of } 9 \\
\text { No participant had a HAS-BLED score of } 6 \text { or } 7 \text {. }\end{array}$} \\
\hline
\end{tabular}
pared with the first 30 days of warfarin treatment, with the adjusted rate for eGFR $<15 \mathrm{ml} / \mathrm{min} / 1.73 \mathrm{~m}^{2}$ more than doubled compared with the referent $(8.3,95 \%$ confidence interval 4.2 to 16.1 per 100 person years $v$ 3.7, 2.7 to 5.0 per 100 person years, respectively; inci4.59; table 2).

Across all eGFR categories, adjusted incidence rates were consistently higher during the first 30 days of warfarin treatment compared with the remainder of follow-up. 


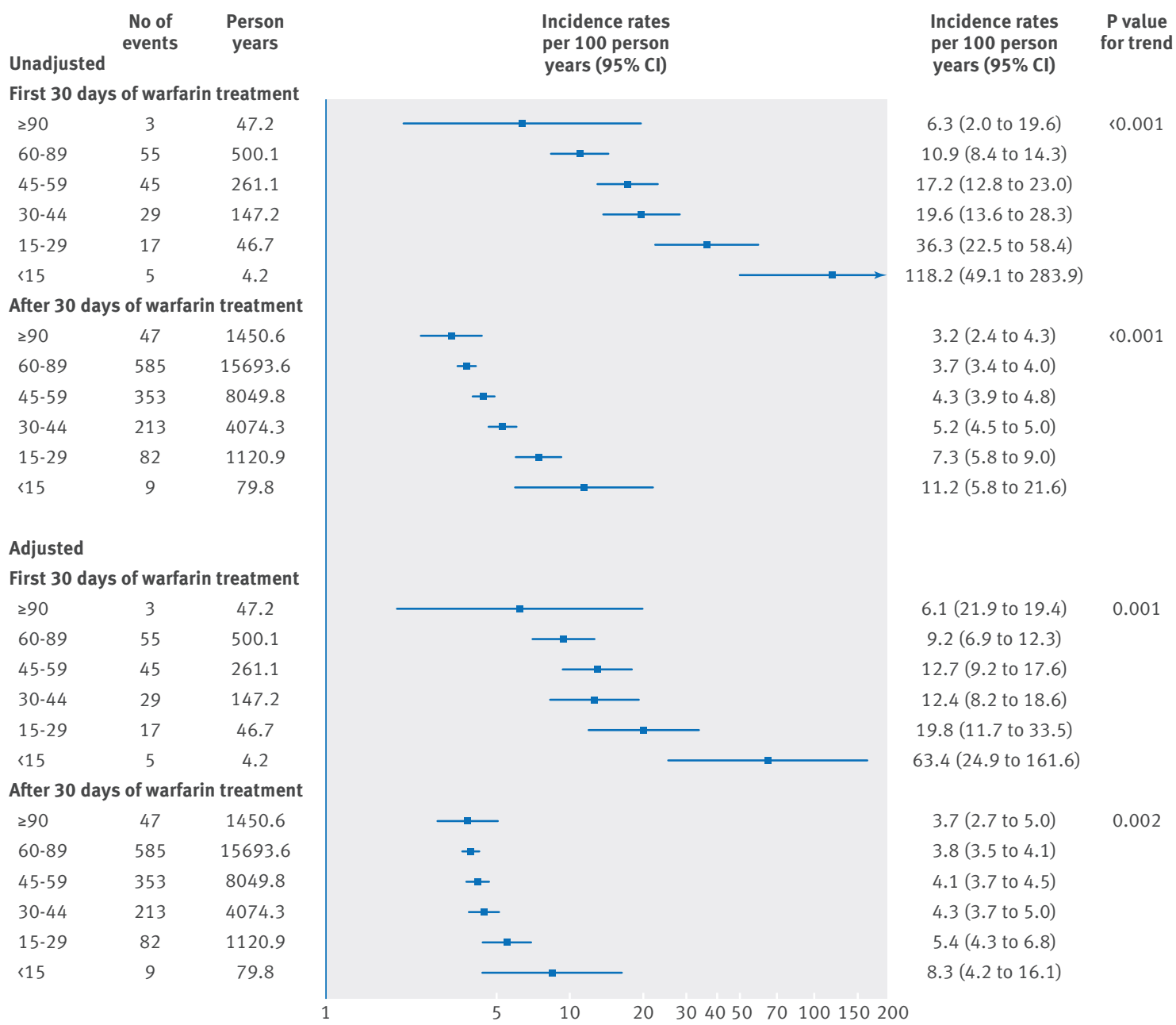

Fig 1 | Unadjusted and adjusted (see footnote to table 2 for adjustment factors) rates per 100 person years of major bleeding by estimated glomerular filtration rate (eGFR) categories

\section{Number needed to treat to harm}

Among participants with chronic kidney disease, the number needed to treat to harm was 22 (95\% confidence interval 18 to 27) during the first 30 days of warfarin treatment, compared with 206 (185 to 231) during the remainder of the study follow-up.

\section{Types of bleeding}

Of the 1443 major bleeding events, $843(58.4 \%)$ were due to a gastrointestinal bleed and $79(5.4 \%)$ to intracranial bleeding. The proportion of the cohort who experienced a gastrointestinal bleeding across the six eGFR categories were, in descending order: 5.6\% (95\% confidence interval 3.9\% to $7.9 \%$ ), $5.5 \%$ (4.9\% to $6.1 \%$ ), $7.3 \%$ (6.5\% to $8.3 \%$ ), $8.4 \%$ (7.2\% to $9.8 \%), 11.6 \%$ (9.1\% to $14.5 \%)$, and $20 \%$ (10.4\% to $32.9 \%$ ), respectively, and for intracranial bleeding were: $0 \%, 0.7 \%(0.6 \%$ to $1.0 \%), 0.5 \%$ ( $0.3 \%$ to $0.9 \%), 0.6 \%$ ( $0.3 \%$ to $0.1 \%), 0.3 \%(0.04 \%$ to $1.2 \%)$, and $0 \%$, respectively (fig 2). Overall, 59 participants (4.0\%) with a bleeding episode died in hospital or within seven days of being discharged. Mortality during this period was highest among participants who experienced intracranial bleeding $(\mathrm{n}=14,17.7 \%)$ compared with gastrointestinal bleeding $(n=35,4.1 \%)$ or other bleeding (1.9\%).
Association between international normalised ratio and eGFR for participants with major bleeding Information on international normalised ratios was available for 491 of the 1443 participants (34.0\%) with a major bleeding episode. In participants who experienced a major bleeding episode, the proportion with an international normalised ratio $>3$ was similar across eGFR categories (31.2\%, 45.9\%, 46.4\%, 45.1\%, 51.7\%, $0 \%$, respectively; Fisher's exact $\mathrm{P}=0.712$ ).

\section{Severity of major bleeding events}

Among the participants who experienced a major bleeding event, 1274 (88.3\%) had one or more haemoglobin measurements during the three day period, with a mean haemoglobin concentration of $104.5 \mathrm{~g} / \mathrm{L}$ (SD $27.3 \mathrm{~g} / \mathrm{L}$ ). Haemoglobin levels were significantly lower in gastrointestinal or other bleeding events compared with intracranial bleeding, and $18.4 \%$ of people had haemoglobin concentrations $<80 \mathrm{~g} / \mathrm{L}$, suggestive of a severe bleed (data not shown). Of the participants who experienced a gastrointestinal bleeding event, $60.0 \%(n=506)$ received one or more endoscopic procedures within seven days after the bleeding event. 


\begin{tabular}{|c|c|c|}
\hline eGFR categories by treatment duration & Incidence rate ratio* $(95 \% \mathrm{Cl})$ & P value \\
\hline \multicolumn{3}{|l|}{ First 30 days of warfarin treatment } \\
\hline \multicolumn{3}{|l|}{$\mathrm{eGFR}\left(\mathrm{mL} / \mathrm{min} / 1.73 \mathrm{~m}^{2}\right)$ : } \\
\hline$\geq 90$ (reference) & 1.00 & - \\
\hline $60-89$ & $1.50(0.46$ to 4.88$)$ & 0.492 \\
\hline $45-59$ & $2.07(0.63$ to 6.83$)$ & 0.228 \\
\hline $30-44$ & $2.02(0.59$ to 6.84$)$ & 0.257 \\
\hline $15-29$ & $3.22(0.90$ to 11.45$)$ & 0.070 \\
\hline$<15$ & $10.33(2.34$ to 45.54$)$ & 0.002 \\
\hline \multicolumn{3}{|l|}{ After 30 days of warfarin treatment } \\
\hline \multicolumn{3}{|l|}{ eGFR $\left(\mathrm{mL} / \mathrm{min} / 1.73 \mathrm{~m}^{2}\right)$ : } \\
\hline$\geq 90$ (reference) & 1.00 & - \\
\hline $60-89$ & $1.03(0.76$ to 1.39$)$ & 0.833 \\
\hline $45-59$ & $1.10(0.80$ to 1.50$)$ & 0.539 \\
\hline $30-44$ & $1.16(0.84$ to 1.62$)$ & 0.352 \\
\hline $15-29$ & $1.45(1.00$ to 2.11$)$ & 0.049 \\
\hline$<15$ & $2.22(1.07$ to 4.59$)$ & 0.031 \\
\hline \multicolumn{3}{|c|}{$\begin{array}{l}\text { *Adjusted for sex, age, First Nations' status, hypertension, diabetes, Charlson comorbidities (cancer, } \\
\text { cerebrovascular disease, congestive heart failure, chronic obstructive pulmonary disease, dementia, metastatic } \\
\text { solid tumour, myocardial infarction, mild liver disease, moderate or severe liver disease, paralysis, peptic ulcer } \\
\text { disease, peripheral vascular disease, rheumatic disease), previous admission to hospital for a bleeding episode } \\
\text { proteinuria, drug use (antiplatelet agents, non-steroidal anti-inflammatory drugs, and proton pump inhibitors), } \\
\text { region, and income. }\end{array}$} \\
\hline
\end{tabular}

Other risk factors for major bleeding

In the first 30 days of warfarin treatment, age and antiplatelet agent use were significantly associated with an increased risk of major bleeding (data not shown). After 30 days of treatment, age, sex, cancer, congestive heart failure, chronic obstructive pulmonary disease, peripheral vascular disease, previous admission to hospital for bleeding, and heavy proteinuria were associated with the risk of major bleeding (data not shown).

\section{Sensitivity analysis}

Our results remained unchanged when eGFR $\geq 60 \mathrm{ml} /$ $\mathrm{min} / 1.73 \mathrm{~m}^{2}$ was used as the reference group, when analyses were repeated excluding participants with major bleeding in the emergency department, and when the study end date for stopping warfarin was defined as the date of the last warfarin prescription after the index date plus 30 days. Participants excluded based on lack
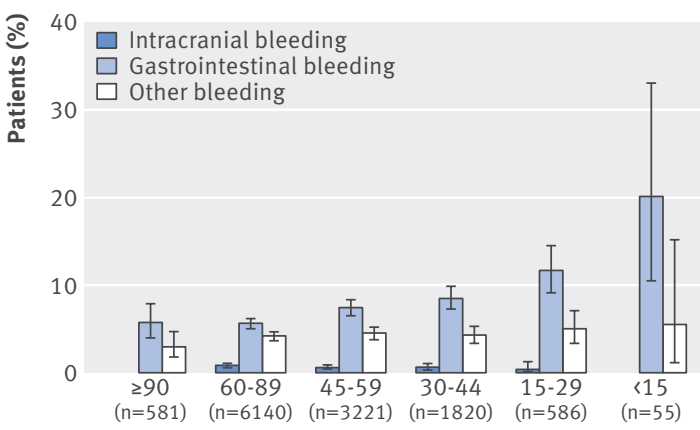

eGFR categories $\left(\mathrm{mL} / \mathrm{min} / 1.73 \mathrm{~m}^{2}\right)$

Fig 2 Percentage of cohort experiencing a major bleeding episode, by type (intracranial bleeding, gastrointestinal bleeding, or other bleeding) and estimated glomerular filtration rate (eGFR). Results represent percentage of cohort experiencing major bleeding over the duration of study follow-up; bars represent $95 \%$ confidence intervals of an outpatient serum creatinine measurements (18\% of participants with atrial fibrillation starting warfarin) were comparable to the study cohort for age, First Nations' status, hypertension, and diabetes; however, the prevalence of a select number of comorbidities such as cancer, congestive heart failure, and rheumatic disease was higher in the study cohort compared with the excluded group of participants (see supplementary table 2). Major bleeding rates for participants with no outpatient serum creatinine measurement available for the estimation of glomerular filtration rate were similar to those with a baseline eGFR of $60-89 \mathrm{ml} / \mathrm{min} / 1.73 \mathrm{~m}^{2}$.

\section{Discussion}

In this population based cohort of older adults with atrial fibrillation starting warfarin treatment, we found that a lower estimated glomerular filtration rate (eGFR) was associated with higher rates of major bleeding. The absolute rates of major bleeding were consistently higher in the first 30 days of warfarin treatment across all levels of eGFR when compared with the remainder of follow-up, with a 10-fold higher rate of major bleeding for the lowest compared with the highest eGFR stratum. The increased risk of bleeding during this period for participants with chronic kidney disease was reflected by a number needed to treat to harm of only 22 (that is, for every 22 people with chronic kidney disease starting warfarin treatment 1 would experience a bleeding episode in the first 30 days of treatment). Subsequently, the association became attenuated, although bleeding rates remained more than double for people in the lowest eGFR category compared with those in the highest, beyond 30 days of warfarin use.

Warfarin is highly effective for preventing embolic stroke due to atrial fibrillation in the general population. A meta-analysis of eight trials (9598 participants) assessed the efficacy of oral anticoagulant treatment, including warfarin, compared with aspirin and reported a $32 \%$ reduction in the risk of stroke with oral anticoagulants. ${ }^{26}$ In contrast, uncertainty remains about the safety of warfarin in people with chronic kidney disease, as warfarin induced bleeding may be of particular concern in this population. Indeed, although the risk of bleeding is relatively modest in the general population, haemostatic dysfunction has been described in people with even mild chronic kidney disease. ${ }^{27-29}$ Deteriorating kidney function and accumulating uraemic toxins contribute to abnormalities in platelet recruitment and activation, which are central to the prevention of bleeding. ${ }^{27-30}$ Thus, although people with chronic kidney disease have higher rates of thromboembolism than the general population, they are also predisposed to an increased risk of bleeding. However, the safety profile of warfarin across the spectrum of chronic kidney disease has not been well defined as most studies to date have focused on patients requiring long term dialysis, with reported highly variable rates of bleeding and limited generalisability to people with less severe chronic kidney disease. ${ }^{8} 3132$ Accordingly, recent international guidelines do not include recommendations on the safety of warfarin in patients with chronic kidney 
disease who do not require dialysis but advise caution when prescribing warfarin to patients with glomerular filtration rates $<30 \mathrm{ml} / \mathrm{min} / 1.73 \mathrm{~m}^{2}{ }^{31}{ }^{33}$ Our study suggests that the risk of warfarin treatment should be carefully weighed against the potential benefits based on the presence of comorbidities and the assessment of the bleeding risk among patients with reduced kidney function (for example, $<60 \mathrm{ml} / \mathrm{min} / 1.73 \mathrm{~m}^{2}$ ), and particularly in those with very reduced kidney function and during the first 30 days of treatment.

\section{Findings in relation to other studies}

Few studies have assessed the risk of major bleeding with warfarin treatment in earlier stages of chronic kidney disease, and our results are consistent with two such studies. ${ }^{1234}$ Although these studies have shown an overall increase in the risk of bleeding in patients with advanced chronic kidney disease, they have been limited by the lack of information on baseline kidney function $^{12}$ and small sample size. ${ }^{34}$

A recent study assessed the bleeding risk associated with warfarin treatment among patients with atrial fibrillation admitted to hospital with myocardial infarction in Sweden. ${ }^{11}$ The authors reported similar unadjusted bleeding rates for patients who received $(\mathrm{n}=5292)$ and did not receive $(\mathrm{n}=19025)$ warfarin at hospital discharge, across all eGFR strata. While bleeding rates increased with declining eGFR, the rates for warfarin users were approximately $50 \%$ lower in the $<15 \mathrm{ml} / \mathrm{min} / 1.73 \mathrm{~m}^{2}$ group than in our study. This might be because almost $70 \%$ of patients who received warfarin at hospital discharge had been receiving warfarin before admission, thus the results are most applicable to prevalent users rather than incident users. Our study adds important new information about the increased risk of bleeding events associated with worsening kidney function in new users of warfarin and highlights the increased early risk, assessments of which were not available from previous studies in patients with atrial fibrillation and chronic kidney disease. Importantly, we assessed the safety of incident warfarin use, which eliminates the biases associated with safety assessments of prevalent drug use. ${ }^{35}$

Our study has important implications for the management of all people with atrial fibrillation and reduced kidney function starting warfarin. This is particularly pertinent in the primary care setting where patients with chronic kidney disease are managed, as we observed rates of major bleeding consistently higher in the first 30 days of warfarin treatment compared with the remainder of the follow-up period, across all levels of eGFR. These findings of increased early bleeding rates are similar to those of a large $(n=125195)$ community based cohort study in Canada of participants with atrial fibrillation starting warfarin, and suggests the safety of warfarin in this patient group may vary depending on kidney function and duration of treatment. ${ }^{13}$

Importantly, we did not observe an increase in the rate of intracranial bleeding (perhaps the most feared complication of warfarin treatment) by eGFR, and the increased likelihood of major bleeding at lower eGFR was primarily driven by differences in the rates of gastrointestinal bleeding. However, it may be possible that an association between intracranial bleeding and kidney function was not observed owing to the limited number of events. None the less, these findings need to be weighed against the potential benefit of warfarin in the prevention of ischaemic stroke, although the efficacy is less certain among patients with chronic kidney disease, particularly those requiring dialysis. ${ }^{36}$ Previous studies have shown that patients who require dialysis have an increased risk of gastrointestinal bleeding compared with matched controls without chronic kidney disease. ${ }^{737}$ Our results indicate that there is a graded and independent association between the likelihood of serious bleeding and the severity of chronic kidney disease.

In recent years, randomised trials assessing the efficacy of novel oral anticoagulant agents in people with atrial fibrillation, including dabigatran, rivaroxaban, and apixaban have shown non-inferiority compared with warfarin. ${ }^{38}$ However, trials have excluded participants with severe renal impairment. Given that these drugs are excreted renally and the lack of treatments to rapidly reverse their anticoagulant effect, these agents may pose a major risk in patients with reduced kidney function. Further assessment of the safety of novel oral anticoagulants in patients with atrial fibrillation and chronic kidney disease is required.

\section{Limitations of this study}

Our study has limitations. We specifically focused on the safety, and not efficacy, of warfarin treatment by kidney function in people with atrial fibrillation. While our results should be weighed against the potential benefit of warfarin treatment in this population, further large scale studies to assess the risk-benefit ratio of warfarin across the spectrum of chronic kidney disease in people with atrial fibrillation are needed. People in Alberta are eligible for drug coverage through the provincial universal healthcare system at 65 years of age, thus participants aged less than 66 years were not included. However, approximately $70 \%$ of people with atrial fibrillation are aged between 65 and 85 years, ${ }^{39}$ and therefore our results are generalisable to most people who would be considered for warfarin to prevent stroke. Although there are advantages to using administrative data in assessing the safety of therapeutic interventions, including the ability to use real world populations, large sample sizes, and low cost, limitations also need to be considered. Of note, we used a validated algorithm to identify atrial fibrillation where the sensitivity and specificity approached 95\% and 99\%, respectively.

Although we adjusted for prescription antiplatelets, non-steroidal anti-inflammatory drugs, and proton pump inhibitors, we did not have information on over the counter drug use, and thus the impact of these drugs may be underestimated. Our study included a large cohort of people with atrial fibrillation, but the number of participants with substantially reduced 
kidney function (that is, $<30 \mathrm{ml} / \mathrm{min} / 1.73 \mathrm{~m}^{2}$ ) compared with those with normal or moderate kidney function, was small, affecting the stability of the point estimates. Owing to the limited availability and irregular frequency of international normalised ratio measurements in the study cohort, we were unable to determine the time patients who experienced a major bleeding event were in the therapeutic range. In addition, it is possible that the lack of an observed association between the proportion of participants with international normalised ratios $>3$ and kidney function was a result of the limited availability of data on international normalised ratios. The prevalence of a select number of comorbidities was higher in the study cohort compared with participants excluded from the study owing to the lack of outpatient serum creatinine measurements. As such, although our study design included a well defined population based cohort of older people with a history of atrial fibrillation and access to universal healthcare including coverage for warfarin treatment, the possibility of selection bias remains. Finally, despite our best efforts to adjust for clinically relevant characteristics, the possibility of residual confounding remains.

\section{Conclusions}

Among older people with atrial fibrillation who started warfarin, rates of major bleeding were significantly higher in those with lower eGFR, particularly during the first 30 days of treatment. The high risk of bleeding in people with chronic kidney disease during the first 30 days was reflected by a needed to treat to harm of 22 compared with 206 in the remainder of the follow-up.

Our results support the need for careful consideration of the bleeding risk relative to kidney function when assessing the risk-benefit ratio of warfarin treatment in people with chronic kidney disease and atrial fibrillation. Given the lack of randomised controlled trial evidence in this patient population, the effectiveness of warfarin treatment remains uncertain, and determination of the risk-benefit ratio is challenging. The use of stroke and bleeding risk stratification schemes such as the $\mathrm{CHADS}_{2}$ and HAS-BLED score may aid in clinical decision making, although this remains unproved. Further large scale studies are needed to assess the effectiveness as well as the balance of harm and benefits of warfarin in patients with atrial fibrillation and reduced kidney function.

Contributors: BRH had full access to all of the data in the study and takes responsibility for the integrity of the data and the accuracy of the data analysis. She is the guarantor. MJ, MT, WCW, and BRH contributed to the study concept and design. MJ, ZM, and BRH contributed to the statistical analyses, and all authors contributed to the analysis and interpretation of the data. MJ and BRH drafted the initial manuscript and all authors contributed to the critical revision of the manuscript.

Funding: MJ was supported by postdoctoral fellowships from the Canadian Institutes of Health Research and Alberta Innovates Health Solutions, and an early career fellowship from the National Health and Medical Research Council of Australia. BRH was supported by the Roy and Vi Baay Chair in Kidney Research; MT was supported by a Canada Research Chair. WCW was supported by grant R01DK095024 from the National Institute for Diabetes and Digestive and Kidney Diseases. WCW enjoys salary and research support through the endowed Gordon A Cain Chair in Nephrology at Baylor College of Medicine. The funding organisations had no role in the design and conduct of the study; in the collection, analysis, and interpretation of the data; or in the preparation, review, or approval of the manuscript.

Competing interests: All authors have completed the ICMJE uniform disclosure form at www.icmje.org/coi disclosure.pdf and declare: MT] received an honorarium for presentation at an industry sponsored conference by Amgen. WCW serves on an event adjudication committee for a prospective study of an arrhythmia device in patients requiring haemodialysis sponsored by Medtronic. This study is based in part by data provided by Alberta Health and Alberta Health Services. The interpretation and conclusions are those of the researchers and do not represent the views of the Government of Alberta.

Ethical approval: This study was approved by the institutional ethics review board of the University of Calgary (REB13-0921). Patient consent was waivered.

Data sharing: Alberta Health and Alberta Health Services does not permit the release of individual participant data or aggregate, summary level data.

Transparency: The lead author (BRH) affirms that the manuscript is an honest, accurate, and transparent account of the study being reported; that no important aspects of the study have been omitted; and that any discrepancies from the study as planned (and, if relevant, registered) have been explained.

This is an Open Access article distributed in accordance with the Creative Commons Attribution Non Commercial (CC BY-NC 4.0) license, which permits others to distribute, remix, adapt, build upon this work non-commercially, and license their derivative works on different terms, provided the original work is properly cited and the use is non-commercial. See: http://creativecommons.org/licenses/ by-nc/4.0/.

1 Go AS, Chertow GM, Fan D, McCulloch CE, Hsu C. Chronic kidney disease and the risks of death, cardiovascular events, and hospitalization. [Published erratum appears in N Engl / Med 2008;18:4.] N Engl J Med 2004:351:1296-305.

2 Smith DH, Gullion CM, Nichols G, Keith DS, Brown JB. Cost of medical care for chronic kidney disease and comorbidity among enrollees in a large HMO population. J Am Soc Nephrol 2004;15:1300-6.

3 United States Renal Data System. 2005 Annual data report: atlas of end-stage renal disease in the United States. USRDS.

4 Ninomiya T, Kiyohara Y, Kubo M, Tanizaki Y, Doi Y, Okubo, et al. Chronic kidney disease and cardiovascular disease in a general Japanese population: the Hisayama Study. Kidney Int 2005;68:228-36.

5 Wolf PA, Abbott RD, Kannel WB. Atrial fibrillation as an independent risk factor for stroke: the Framingham Study. Stroke 1991;22:983-8.

6 Holden RM, Harman GJ, Wang M, Holland D, Day AG. Major bleeding in hemodialysis patients. Clin J Am Soc Nephrol 2008;3:105-110.

7 Luo JC, Leu HB, Hou MC, Huang KW, Lin HC, Lee FY, et al. Nonpeptic ulcer, nonvariceal gastrointestinal bleeding in hemodialysis patients. Am J Med 2013;126:264.e225-32.

8 Winkelmayer WC, Liu J, Setoguchi S, Choudhry NK. Effectiveness and safety of warfarin initiation in older hemodialysis patients with incident atrial fibrillation. Clin I Am Soc Nephrol 2011:6:2662-8.

9 Hohnloser SH, Hijazi Z, Thomas L, Alexander JH, Amerena J, Hanna M, et al. Efficacy of apixaban when compared with warfarin in relation to renal function in patients with atrial fibrillation: insights from the ARISTOTLE trial. Eur Heart J 2012;33:2821-30.

10 Hijazi Z, Hohnloser SH, Oldgren J, Andersson U, Connolly SJ, Eikelboom JW, et al. Efficacy and safety of dabigatran compared with warfarin in relation to baseline renal function in patients with atrial fibrillation: a RE-LY (Randomized Evaluation of Long-term Anticoagulation Therapy) trial analysis. Circulation 2014;129:961-70.

11 Carrero IJ, Evans M, Szummer K, Spaak J, Lindhagen L, Edfors R, et al. Warfarin, kidney dysfunction, and outcomes following acute myocardial infarction in patients with atrial fibrillation. JAMA 2014:311:919-28.

12 Olesen JB, Lip GY, Kamper AL, Hommel K, Kober L, Lane DA, et al. Stroke and bleeding in atrial fibrillation with chronic kidney disease. [Published erratum appears in N Engl J Med 2012;367:2262.] N Engl] Med 2012:367:625-35.

13 Gomes T, Mamdani MM, Holbrook AM, Paterson M, Hellings C, Juurlink DN. Rates of hemorrhage during warfarin therapy for atrial fibrillation. CMAI 2013;185:E121-7.

14 Hemmelgarn BR, Clement F, Manns BJ, Klarenbach S, James MT, Ravani P, et al. Overview of the Alberta Kidney Disease Network. BMC Nephrol 2009;10:30

15 Borzecki AM, Wong AT, Hickey EC, Ash AS, Berlowitz DR. Identifying hypertension-related comorbidities from administrative data: what's the optimal approach? Am J Med Qual 2004;19:201-6.

16 Dublin S, French B, Glazer NL, Wiggins KL, Lumley T, Psaty BM, et al. Risk of new-onset atrial fibrillation in relation to body mass index. Arch Intern Med 2006;166:2322-8.

17 Jensen PN, Johnson K, Floyd J, Heckbert SR, Carnahan R, Dublin S. A systematic review of validated methods for identifying atrial 
fibrillation using administrative data. Pharmacoepidemiol Drug Saf 2012;21(Suppl 1):141-7.

18 Levey AS, Stevens LA, Schmid CH, Zhang YL, Castro AF III, Feldman HI, et al. A new equation to estimate glomerular filtration rate. [Published erratum appears in Ann Intern Med 2011;155:408.] Ann Intern Med 2009;150:604-12.

19 Kidney Disease: Improving Global Outcomes (KDIGO) CKD Work Group. KDIGO 2012 Clinical practice guideline for the evaluation and management of chronic kidney disease. Kidney Int 2013;(Suppl 3):1-150

20 Statistics Canada. Ethnocultural portrait of Canada highlight tables, 2006 census. www12.statcan.ca/english/census06/data/highlights/ ethnic/index/cfm?Lang $=\mathrm{E}$

21 Hux JE, Ivis F, Flintoft V, Bica A. Diabetes in Ontario: determination of prevalence and incidence using a validated administrative data algorithm. Diabetes Care 2002;25:512-6.

22 Quan H, Khan N, Hemmelgarn BR, Tu K, Chen G, Campbell B, et al. Validation of a case definition to define hypertension using administrative data. Hypertension 2009;54:1423-8

23 Gage BF Waterman AD, Shannon W, Boechler M, Rich MW, Radford M). Validation of clinical classification schemes for predicting stroke: results from the National Registry of Atrial Fibrillation. JAMA 2001;285:2864-70

24 Quan H, Sundararajan V, Halfon P, Fong A, Burnand B, Luthi JC, et al. Coding algorithms for defining comorbidities in ICD-9-CM and ICD-10 administrative data. Med Care 2005;43:1130-9.

25 Arnason T, Wells PS, van Walraven C, Forster AJ. Accuracy of coding for possible warfarin complications in hospital discharge abstracts. Thromb Res 2006;118:253-62.

26 Aguilar MI, Hart R, Pearce LA. Oral anticoagulants versus antiplatelet therapy for preventing stroke in patients with non-valvular atrial fibrillation and no history of stroke or transient ischemic attacks. Cochrane Database Syst Rev 2007;3:CD006186.

27 Jalal DI, Chonchol M, Targher G. Disorders of hemostasis associated with chronic kidney disease. Sem Thromb Hemost 2010;36:34-40.

28 Pavord S, Myers B, Pavord S, Myers B. Bleeding and thrombotic complications of kidney disease. Blood Rev 2011:25:271-8.
29 Thijs A, Nanayakkara PW, Ter Wee PM, Huijgens PC, van Guldener C, Stehouwer CD. Mild-to-moderate renal impairment is associated with platelet activation: a cross-sectional study. Clin Nephrol 2008:70:325-31

$30 \mathrm{Ng} \mathrm{KP}$, Edwards NC, Lip GY, Townend JN, Ferro C). Atrial fibrillation in CKD: balancing the risks and benefits of anticoagulation. Am J Kidney Dis 2013;62:615-32.

31 Chan KE, Lazarus JM, Thadhani R, Hakim RM. Warfarin use associates with increased risk for stroke in hemodialysis patients with atrial fibrillation. I Am Soc Nephrol 2009:20:2223-33.

32 Mokrzycki MH, Jean-Jerome K, Rush H, Zdunek MP, Rosenberg SO A randomized trial of minidose warfarin for the prevention of late malfunction in tunneled, cuffed hemodialysis catheters. Kidney Int 2001:59:1935-42.

33 Bennett WM, Bennett WM. Should dialysis patients ever receive warfarin and for what reasons? Clin J Am Soc Nephrol 2006;1:1357-9.

34 Limdi NA, Beasley TM, Baird MF, Goldstein JA, McGwin G, Arnett DK, et al. Kidney function influences warfarin responsiveness and hemorrhagic complications. J Am Soc Nephrol 2009;20:912-21.

35 Ray WA, Ray WA. Evaluating medication effects outside of clinical trials: new-user designs. Am J Epidemiol 2003;158:915-20.

36 Shah M, Tsadok MA, Jackevicius CA, Essebag V, Eisenberg MJ, Rahme $\mathrm{E}$, et al. Warfarin use and the risk of stroke and bleeding in patients with atrial fibrillation undergoing dialysis. Circulation 2014;129:1196-203

37 Kuo CC, Kuo HW, Lee IM, Lee CT, Yang CY. The risk of upper gastrointestinal bleeding in patients treated with hemodialysis: a population-based cohort study. BMC Nephrol 2013;14:15.

38 Harel Z, Sholzberg M, Shah PS, Pavenski K, Harel S, Wald R, et al. Comparisons between novel oral anticoagulants and vitamin $\mathrm{K}$ antagonists in patients with CKD. J Am Soc Nephrol 2014:25:431-42.

39 Feinberg WM, Blackshear JL, Laupacis A, Kronmal R, Hart RG. Prevalence, age distribution, and gender of patients with atrial fibrillation. Analysis and implications. Arch Intern Med 1995;155:469-73.

(c) BMJ Publishing Group Ltd 2015 